International Journal of Molecular Medicine and Advance Sciences 7 (3): 12-15, 2011

ISSN: $1813-176 \mathrm{X}$

(C) Medwell Journals, 2011

\title{
Clinical Correlates Involving Deficiency of Vitamin D and Calcium
}

\author{
Stephanie Galea and Renald Blundell \\ Department of Physiology and Biochemistry, University of Malta, Msida, Malta
}

\begin{abstract}
The effects of vitamin D and calcium deficiency are evident in several patients suffering from various diseases including osteoporosis and rickets. Other hormones such as glucocorticoids also play a role in bone homeostasis leading to bone growth promotion or retardation. Consequently, hormone imbalances in patients should be considered with suspected vitamin D and calcium deficiency. The deficiency of vitamin D and calcium lead to non-bone related abnormalities for instance promotion of prostate cancer and retardation of blood clotting, respectively. However, this study will be focusing on the detrimental effects of osteoporosis and rickets on patients. Vitamin D and calcium deficiency may be prevented by adequate sun exposure as well as diet supplementation. Vitamin D and calcium deficiency result in lack of intracellular mechanisms, loss of bone matrix and density hence, weaker bones. This leads to an increased probability of bone fractures.
\end{abstract}

Key words: Vitamin D, calcium, osteoporosis, rickets, diet, deficiency

\section{INTRODUCTION}

Several diseases such as osteoporosis, rickets, osteomalacia and rheumatoid arthritis have been linked with problems due to bone metabolism, often involving deficiency of vitamin $\mathrm{D}$ and calcium. The recommended daily dietary intake of vitamin D is $600 \mathrm{IU}$, ideally increased to $800 \mathrm{IU}$ in the elderly, apart from having adequate exposure to UV light (http://ods.od.nih.gov/ factsheets/vitamind). Vitamin D rich foods include oily fish, milk and eggs as mentioned previously. Calcium rich sources include milk, cheese and yoghurt and the recommended daily intake is shown in the Table 1 . According to Dowd and Stafford (2008), ideal vitamin D levels should be between 50 and 70 and additional calcium supplementation is unnecessary if the diet is acid-base balanced. For instance in osteoarthritis, the disease in bone remodelling occurs due to vitamin deficiency and acid excess in the diet. Therefore, calcium supplementation would be unlikely to improve the prognosis of the patient.

Calcium is absorbed in the small intestine via proteins such as TRPV6, calbindin 9K, PMCA1 and NCX1 and a small percentage remains in the intestines to be egested in the stools. Since, vitamin D is lipid soluble when obtained through the diet it diffuses through intestinal epithelium. Until recently, it was thought that people suffering from Crohn's disease and coeliac disease have impaired calcium and vitamin D uptake (Douard et al., 2010). This would increase the risks having of bone fractures, rickets in growing children and osteoporosis. However, in a study shown by Kumari et al. (2010), it was found that young men having stable Crohn's disease do
Table 1: A table showing the recommended daily amount of calcium in the diet

\begin{tabular}{lcccc}
\hline Age & Male (mg) & Female (mg) & Pregnant (mg) Lactating (mg) \\
\hline $0-6$ months $^{*}$ & 200 & 200 & - & - \\
7-12 months* & 260 & 260 & - & - \\
1-3 years & 700 & 700 & - & - \\
4-8 years & 1,000 & 1,000 & - & - \\
9-13 years & 1,300 & 1,300 & - & - \\
14-18 years & 1,300 & 1,300 & 1,300 & 1,300 \\
19-50 years & 1,000 & 1,000 & 1,000 & 1,000 \\
51-70 years & 1,000 & 1,200 & - & - \\
$71+$ years & 1,200 & 1,200 & - & - \\
\hline
\end{tabular}

*Adequate Intake (AI)

not have impaired calcium uptake. It was only in patients who had inflammatory Crohn's disease that calcium uptake was impaired and this problem was solved by administering vitamin $\mathrm{D}$ as it has been suggested to increase calcium transporting proteins and decrease the inflammatory response. This treatment was also found to reduce bone turnover markers and TNF- $\alpha$ levels.

\section{THE ROLE OF OTHER HORMONES IN MAINTAINING BONE HOMEOSTASIS}

Disorders in Growth Hormone (GH), glucocorticoids, Insulin-like Growth Factors (IGFs), thyroid hormones and steroids (such as oestrogens) also affect bone growth. For instance, glucocorticoids given to children may stunt bone growth. Receptors for glucocorticoids are found on osteoblasts and so it seems to be important in bone formation and mineral deposition. However, it was discovered that when given to mice in pharmacological

Corresponding Author: Renald Blundell, Department of Physiology and Biochemistry, University of Malta, Msida, Malta 


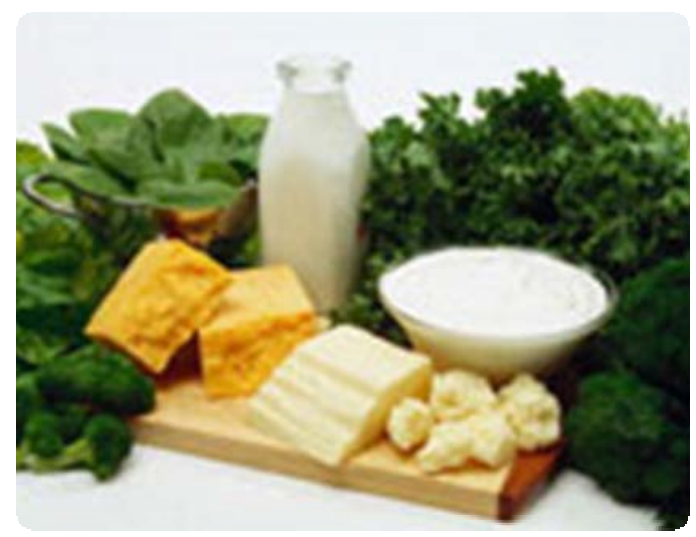

Fig. 1: Foods rich in calcium

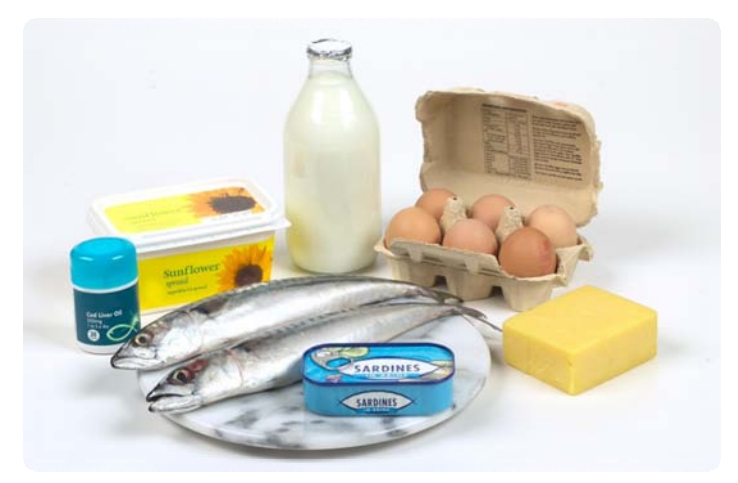

Fig. 2: Foods rich in vitamin $\mathrm{D}$

doses chronically, apoptosis of osteoblasts and osteocytes and inhibition of osteoblast proliferation occurs resulting in osteoporosis (Karaplis, 2008).

Table 1 shows that there is the highest requirement for calcium intake from 9-18 years of age. Pregnant and lactating females do not seem to require a higher calcium intake than that which is considered normal for their age group. Post-menopausal females need a higher calcium intake than males due to their higher risk of developing osteoporosis (http://ods.od.nih.gov/factsheets/calcium).

These foods should be included in people's diets in order to avoid calcium and vitamin $\mathrm{D}$ deficiency. Calcium rich foods include milk, various cheeses, yoghurts and green leafy vegetables (Fig. 1). Vitamin D rich foods include milk, eggs, butter, cheese, cod liver oil, oily fish and sardines as shown in Fig. 2 (http://www. newrinkles.com/index.php/nutrition/calcium-rich-foodsversus-supplements, http://www.cheapethniceatz.com/ 2009/1 1/02/fight-h1n1-with-food/).

\section{OSTEOPOROSIS}

The risk factors of developing osteoporosis include being a post-menopausal elderly female, smoking, lack of exercise being caucasian and frail due to a poor diet and most likely deficient in calcium and vitamin D. Effective calcium and vitamin D supplementation and weightbearing exercises can help prevent osteoporosis. However, in certain cases, osteoporosis can begin in utero if the mother is vitamin D deficient. Osteoporosis affects the inorganic component of bone and results in thinning of bone.

Consequently, bone loses its strength and so is easily fractured. The typical clinical scenario would be an old lady falling and fracturing her hip and neck of femur. More often than not, patients would be unaware of their bone loss (in post-menopausal women it is about 1\% bone loss per year) which is why osteoporosis is referred to as a silent disease.

It develops due to a decrease in oestrogen levels in women. However, it also occurs in men due to a decline in bone density as the body ages (Dowd and Stafford, 2008; Sunyecz, 2008).

In order to minimize risks of developing osteoporosis, it is recommended that exercise, calcium and vitamin D supplementation is taken before the age of 30 as that time would be peak bone mass after which a decline in bone density is observed. Vitamin D deficiency enhances osteoclast proliferation and so increased calcium mobilisation from bone.

It is important to note that calcium supplements may interfere with drugs the patient is taking for instance calcium will increase digoxin to toxicity levels causing arrhythmia and palpitations. All people above the age of 50 should take a bone density test (dual-energy X-ray absorptiometry implementing the $\mathrm{T}$-score or the $\mathrm{Z}$-score) so as to prevent the risk of bone fractures by starting treatment early on. Pharmacological treatment may be given by using bisphosphonates such as reclast and actonel which act by killing off osteoclasts and effectively inhibiting further bone resorption (Sunyecz, 2008).

Osteoporosis causes the spaces between one vertebra and the next to decrease, often resulting in compressional fractures. The patient usually suffers from kyphosis and appears shorter in size because of the compressed vertebrae as shown in Fig. 3 (http://www. true-beauty-tips.com/osteoporosis-alternatives.html).

Osteoporosis is a condition in which lack of calcium and vitamin $\mathrm{D}$ and age, amongst other factors induce a higher rate of bone resorption than bone formation. Consequently, the bone matrix is destroyed, cortical bone thins and larger spaces are formed within the bone architecture as shown in Fig. 4. Bones also appear thinner on X-Rays of osteoporotic patients (http://www. chiropractic-help.com/Causes-of-Osteoporosis.html). 


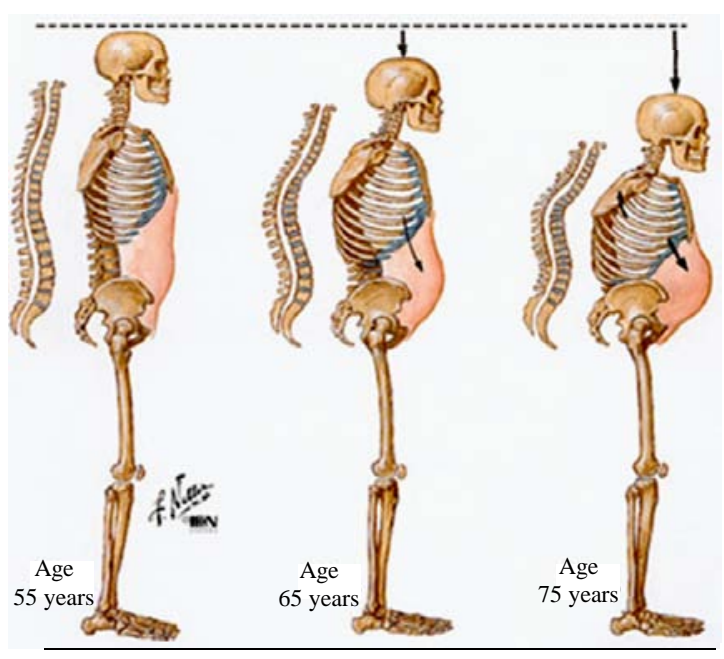

Fig. 3: The progression of osteoporosis after 55 years of age

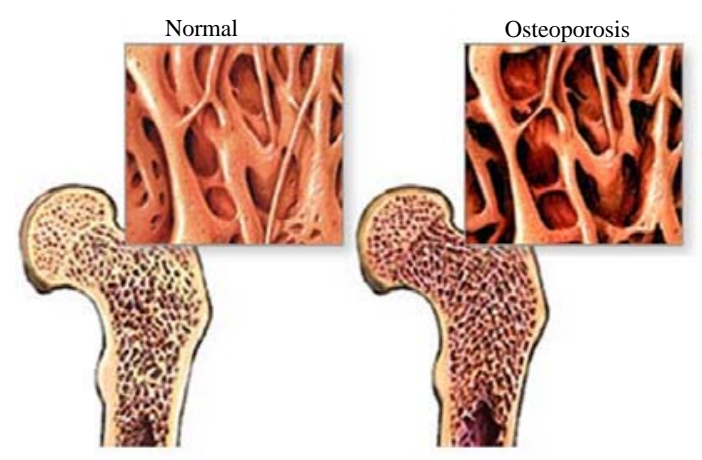

*ADAM

Fig. 4: Cross-section of bone showing increased spaces within trabecular bone and thinning of bone

\section{RICKETS}

This disease manifests in children who are vitamin D deficient and is known as osteomalacia when it occurs in adults often leading to osteoporosis which is a decrease of total bone mass including collagenous tissue in the bone matrix. In the advanced stages, rickets is characterized by growth retardation, epiphysis enlargement in long bones, abnormal projections from the ribcage and bowing or bending of the legs and spine with weak, toneless muscles. This can be reversed by supplementation of high doses vitamin D or calcitriol and ensuring a calcium and phosphate rich diet to replenish the bone reservoir of ions required for homeostasis (Holick, 2006).

Since, vitamin D is deficient in most cases, calcium cannot be absorbed from the intestines to be utilized by bone. The decrease in calcium plasma levels causes an increase in PTH secretion which in turn enhances RANKL on osteoblasts resulting in increased resorption by osteoclasts. Also, phosphorous reabsorption in inhibited in the kidneys by PTH causing increased loss of phosphate in the urine. Since, serum calcium concentrations are also decreased, there is not enough calcium and phosphorous to ensure laying down of bone by osteoblasts.

Also, 1, 25- $(\mathrm{OH})_{2} \mathrm{D}_{3}$ levels are usually normal or high in these children since, excess PTH stimulates its production in the kidneys and so it may appear that the child is not deficient for vitamin D. Coloured peopled were found more likely to be deficient in vitamin $\mathrm{D}$ when compared to their white counterparts. Since, rickets causes poor mineralization of the whole skeleton, bones which grow rapidly such as long bones show the first signs of abnormality especially in infants $<18$ months of age (Holick, 2006).

It is essential that people are exposed to enough sunlight as many people are deficient for vitamin D even though they live in a country with plenty of sunlight. However, this together with an appropriate diet is still not enough for some people because they are genetically vitamin D resistant which leads to rickets. This occurs because a point mutation can occur causing a variation in splicing of VDR by leaving out exon 8, rendering VDR non-functional (Ma et al., 2009) or due to a mutation in an enzyme converting $25-(\mathrm{OH}) \mathrm{D}_{3}$ to $1,25-(\mathrm{OH})_{2} \mathrm{D}_{3}$. The signs for this condition are aggravated when compared to vitamin $\mathrm{D}$ deficiency. The treatment for this condition is pharmacological doses of calcitriol, calcium and phosphorous (Pettifor, 2005).

This X-ray was taken after a 10 months old, coloured boy sustained minor trauma to his left femur. The Fig. 5 shows bowing of the shaft of femur and an incomplete fracture. He was clearly suffering from vitamin D deficiency either due to a primary cause (inadequate diet and lack of sun exposure) or a secondary cause such as chronic renal failure, hypophosphataemia or vitamindependent rickets.

The bowing and fracture occurred because of lack of calcium in the bones causing the bones to be weak and unable to support the patient's body. As a result, minor trauma will cause bones to fracture easily since little resistance is offered to stress (http://www.radpod.org/ 2007/01/08/rickets/).

The Fig. 6 shows the classical appearance of a severe case of rickets including bowed legs, pigeon chest, wide joints, ribs and spinal deformities (http://lancastria.net/ blog/tag/starvation). 


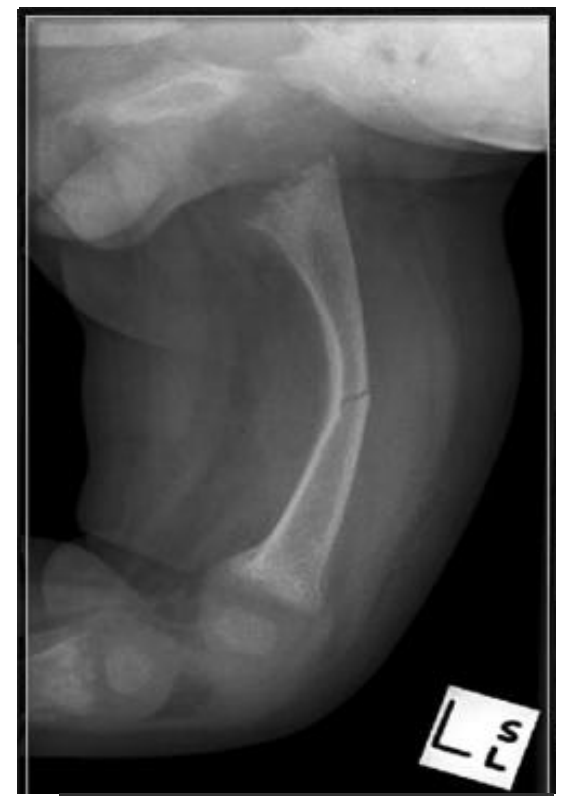

Fig. 5: A radiograph showing left femur bowing and an incomplete fracture

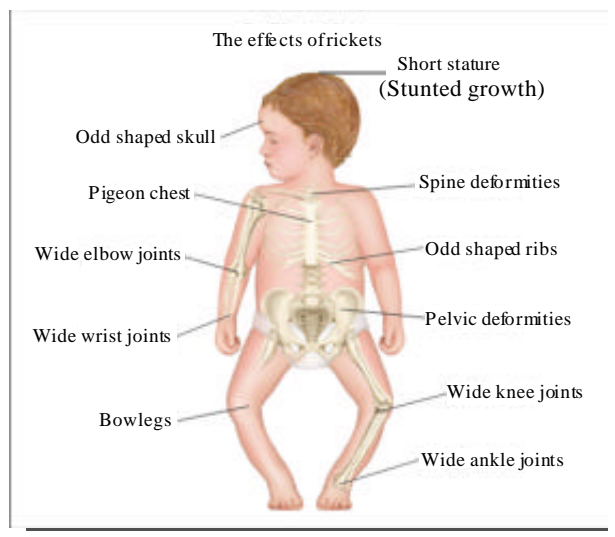

Fig. 6: The clinical signs and symptoms of rickets in a child

\section{CONCLUSION}

It is remarkable how many people throughout the world are deficient for both vitamin D and calcium without themselves knowing. The best way to reach out to people is by educating the required daily amounts for vitamin $\mathrm{D}$ and calcium and showing them the reasons why they are an essential part of the diet especially in regions of the world lacking sunlight. Research is continually unfolding mysteries of the etiology of major diseases for instance prostate cancer and colorectal cancer, several of which

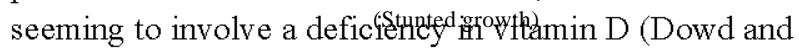
Stafford, 2008). This further stresses the importance of vitamin $\mathrm{D}$ and calcium in not only bone but the entire body's physiology.

\section{REFERENCES}

Douard, V., A. Asgerally, Y. Sabbagh, S. Sugiura, S.A. Shapses, D. Casirola and R.P. Ferrarism, 2010. Dietary fructose inhibits intestinal calcium absorption and induces vitamin D insufficiency in CKD. J. Am. Soc. Nephrol., 21: 261-271.

Dowd, J.E. and D. Stafford, 2008. The Vitamin D Cure. John Wiley and Sons Inc., New Jersey, USA.

Holick, M.F., 2006. Resurrection of vitamin D deficiency and rickets. J. Clin. Invest., 116: 2062-2072.

Karaplis, A.C., 2008. Embryonic Development of Bone and Regulation of Intramembranous and Endochondral Bone Formation. In: Principles of Bone Biology, TwoVolume Set, Volumes 1-2, Bilezikian, J.P., L.G. Raisz and T.J. Martin (Eds.). 3rd Edn., Academic Press Inc., New York, USA., ISBN-13: 9780123738844, pp: 66-76.

Kumari, M., N.B. Khazai, T.R. Ziegler, M.S. Nanes, S.A. Abrams and V. Tangpricha, 2010. Vitamin Dmediated calcium absorption in patients with clinically stable Crohn's disease: A pilot study. Mol. Nutr. Food Res., 54: 1085-1091.

Ma, N.S., P.J. Malloy, P. Pitukcheewanont, D. Dreimane, M.E. Geffner and D. Feldman, 2009. Hereditary vitamin D resistant rickets: Identification of a novel splice site mutation in the vitamin $\mathrm{D}$ receptor gene and successful treatment with oral calcium therapy. Bone, 45: 743-746.

Pettifor, J.M., 2005. Vitamin D Deficiency and Nutritional Rickets in Children. In: Vitamin D, Feldman, D., F.H. Glorieux and J.W. Pike (Eds.). 2nd Edn., Elsevier Academic Press, Philadelphia, USA., ISBN: 0122526872 .

Sunyecz, J.A., 2008. The use of calcium and vitamin D in the management of osteoporosis. Ther. Clin. Risk Manage., 4: 827-836. 